\title{
Cutaneous saltation within and across arms: A new measure of the saltation illusion in somatosensation
}

\author{
MARTIN EIMER and BETTINA FORSTER \\ Birkbeck College, University of London, London, England \\ and \\ JONAS VIBELL \\ University of Oxford, Oxford, England
}

\begin{abstract}
A new objective procedure was used to measure the strength of cutaneous saltation, in order to clarify current debates about the nature of this illusion. Three taps were presented successively to three possible forearm locations. Participants attended to the middle location and reported whether a tap was perceived there. When all stimuli were delivered to the same arm and intertap intervals were short, participants were unable to distinguish real and illusory stimuli at the middle location. When both arms were stimulated, location judgments on one arm were shifted toward a tap subsequently delivered to the other arm. These results challenge the view that saltation is a purely attentional phenomenon, but they are inconsistent with the idea that this illusion is produced in the primary somatosensory cortex.
\end{abstract}

Our conscious perception of sensory stimulation can be strongly biased by the arrival of subsequent events. For example, when a series of tactile stimuli are presented in rapid succession to different locations, perceived locations are shifted toward subsequent stimuli. This robust illusion, termed cutaneous saltation, can be produced when presenting five taps close to the wrist, followed by five taps at the center of the forearm, and another five taps close to the elbow (Geldard \& Sherrick, 1972). Instead of these taps being perceived at their actual locations, they are felt to be uniformly distributed, as if a rabbit were hopping along the arm. This cutaneous rabbit illusion is also present when two successive taps are delivered at one location, followed by a third at another location. The second tap is mislocalized, and the size of its illusory displacement toward the third tap depends on the interstimulus interval (ISI) separating these stimuli (Cholewiak \& Collins, 2000; Geldard, 1975, 1982; Kilgard \& Merzenich, 1995).

The cutaneous rabbit illusion has provoked heated philosophical debates about the timing of subjective experience, since it seems to suggest that conscious perception is influenced by stimuli that have not yet occurred (cf. Dennett, 1991). At the same time, experimental psychologists and neuroscientists have tried to identify the mechanisms responsible for sensory saltation. When researchers mapped the area on the body surface where

This research was supported by a grant from the Biotechnology and Biological Sciences Research Council. M.E. holds a Royal SocietyWolfson Research Merit Award. Correspondence should be addressed to M. Eimer, Department of Psychology, Birkbeck College, University of London, Malet Street, London WC1E 7HX, England (e-mail: m.eimer@, bbk.ac.uk) saltation can be elicited, the cutaneous rabbit was found to be constrained by the anatomical organization of the somatosensory system (Geldard, 1982; Geldard \& Sherrick, 1983). Saltation on the limbs was more pronounced in the longitudinal direction (e.g., from elbow to wrist) than laterally and was eliminated altogether when successive stimuli were delivered to nonadjacent dermatomes, or to the left and right of the body midline.

These findings have led to the proposal that the saltation illusion is produced by strictly local (dermatome-based) interactions between metastable tactile representations at early stages of perceptual processing in the primary somatosensory cortex (Cholewiak, 1999; Geldard, 1975, 1982). However, Kilgard and Merzenich (1995) have challenged the view that sensory saltation is a perceptual illusion. These authors asked participants to attend to proximal or distal regions of the forearm and found that perceived loci of tactile events were systematically shifted toward attended locations. On the basis of these observations, Kilgard and Merzenich argued that saltation reflects a postperceptual attentional bias, resulting from participants attending to predictable locations of anticipated tactile events.

In previous investigations of sensory saltation, participants were typically instructed to point to the perceived location of a tactile event or to adjust the interval between successive taps in order to produce a saltation illusion (psychophysical method of production; Geldard, 1975). The use of subjective location judgments has made it difficult to objectively quantify the strength of this illusion and to investigate how its strength is affected by manipulations of temporal, spatial, or attentional factors. This lack of an objective quantitative measure of saltation may 
help explain why debates with respect to the mechanisms underlying cutaneous saltation remain unresolved. The purpose of the present experiments was to develop and test a new objective measure of the saltation illusion and to use this measure to shed new light on current controversies about the nature of cutaneous saltation.

We developed a novel procedure to measure the presence and strength of the saltation illusion while manipulating temporal parameters of tactile stimulation, stimulation locations, and body posture. On each trial, a sequence of three taps (T1, T2, T3) was presented successively to three possible forearm locations (L1, L2, L3). Participants were instructed to attend to the middle location (L2) and to report at the end of each trial whether or not they had felt a tap at that location. The dependent variable was the number of taps reported to be present at L2 for different types of trials. In Experiment 1, three trial types were compared. On tap trials, all three locations (including L2) were successively stimulated, and present responses were correct. On rabbit trials, T1 and T2 at one outer location (L1 or L3) were followed by T3 at the opposite outer location (L3 or L1); this should result in an illusory displacement of T2 toward L2. Thus, participants should be likely to incorrectly report the presence of a tap at L2. In control trials, all three taps were successively delivered to one of the two outer locations (L1 or L3). Experiment 2 also contained a different type of control trial (motion control), where T1 was delivered at one outer location and T2 as well as T3 at the opposite outer location. According to Geldard (1975, 1982), cutaneous saltation should be absent in both types of control trials, and the number of taps incorrectly reported at L2 should be low.

On the basis of the assumption that the strength of the saltation illusion is reflected by the frequency of taps mislocated at L2 on rabbit trials, the presence and strength of saltation were quantified as follows. First, the number of taps correctly perceived at L2 on tap trials was compared with the number of taps incorrectly localized at L2 on rabbit trials. Identical perceptual reports on these two types of trials (i.e., the absence of significant differences in the percentage of taps reported to be present at L2 on tap and rabbit trials) would suggest that participants are unable to discriminate between real and illusory tap locations (see Cholewiak \& Collins, 2000, for an analogous argument). The question of whether real and illusory percepts are phenomenologically indistinguishable was further explored in Experiment 3 with the use of a two-alternative forced choice procedure. Also, and equally important, the percentage of taps incorrectly reported at L2 was compared for rabbit and control trials. The presence of a cutaneous saltation illusion was inferred whenever a significantly higher percentage of taps was incorrectly reported to be present at L2 on rabbit trials than on control trials.

This general procedure was employed to study two central questions related to the mechanisms responsible for cutaneous saltation. First, the fact that participants were instructed to continuously focus their attention on L2, in order to detect and report any tactile events delivered to this location, allowed us to reinvestigate the claim that the saltation illusion is exclusively determined by expectations and attention (Kilgard \& Merzenich, 1995). If saltation was linked to the current focus of spatial attention, illusory displacements of T2 should occur equally often on rabbit trials and on control trials. With attention constantly focused on L2 in these two types of trials, the perceived location of $\mathrm{T} 2$ will be subject to the same anticipatory spatial bias. Therefore, the finding that taps are more likely to be mistakenly located at L2 on rabbit trials than on control trials would suggest that the saltation illusion remains present even when the attentional factors identified by Kilgard and Merzenich are neutralized.

Second, we reevaluated the hypothesis that the body midline represents an uncrossable boundary for cutaneous saltation by investigating whether this illusion remains present when tactile events are successively delivered to opposite arms. Previous studies reporting that saltation is confined to one side of the body (e.g., Geldard, 1982) have investigated body sites such as left and right forehead or left and right anterior thorax. These areas do not include joints and therefore cannot be independently repositioned with respect to each other. It is possible that saltation will be elicited across the body midline when successive tactile stimuli are delivered to left and right body parts that move separately in external space and can be independently realigned relative to each other and to the body midline. To investigate this possibility, we applied the general procedures described above under conditions where successive taps were delivered to opposite arms, with both arms positioned in parallel and perpendicular to the main body axis (Figure 1B).

\section{EXPERIMENT 1}

Experiment 1 contained two conditions, delivered in separate successive blocks. In the single-arm condition, all taps were delivered to the same arm (Figure 1A). Previous studies (Cholewiak, 1976; Geldard, 1975) have demonstrated that illusory sensations of T2 at L1 as being located midway between L1 and L3 can be most reliably observed for T2-T3 ISIs in the range of 70-150 msec. In order to evaluate the validity of our new measure of saltation, T2-T3 ISIs were set to either $100 \mathrm{msec}$ (where the saltation illusion should be strongest), $200 \mathrm{msec}$ (where the strength of this illusion should be reduced), and $400 \mathrm{msec}$ (where the illusion should be largely absent). Unlike in most previous investigations of saltation, where the T1-T2 ISI was typically longer than that separating T2 and T3, T1-T2 ISIs were identical to T2-T3 ISIs in Experiment 1. This was done to preclude the possibility that perceptual reports of T2 locations would be influenced by the tau effect (Helson \& King, 1931). When successive tactile events are separated by unequal intervals, their perceived spatial separation can be biased, with stimuli separated by short intervals judged to be closer together than those separated by longer intervals. ${ }^{1}$

The both-arms condition was included so that we could reinvestigate the claim that the cutaneous rabbit is unable to cross the body midline. Here, L1 and L2 were located 

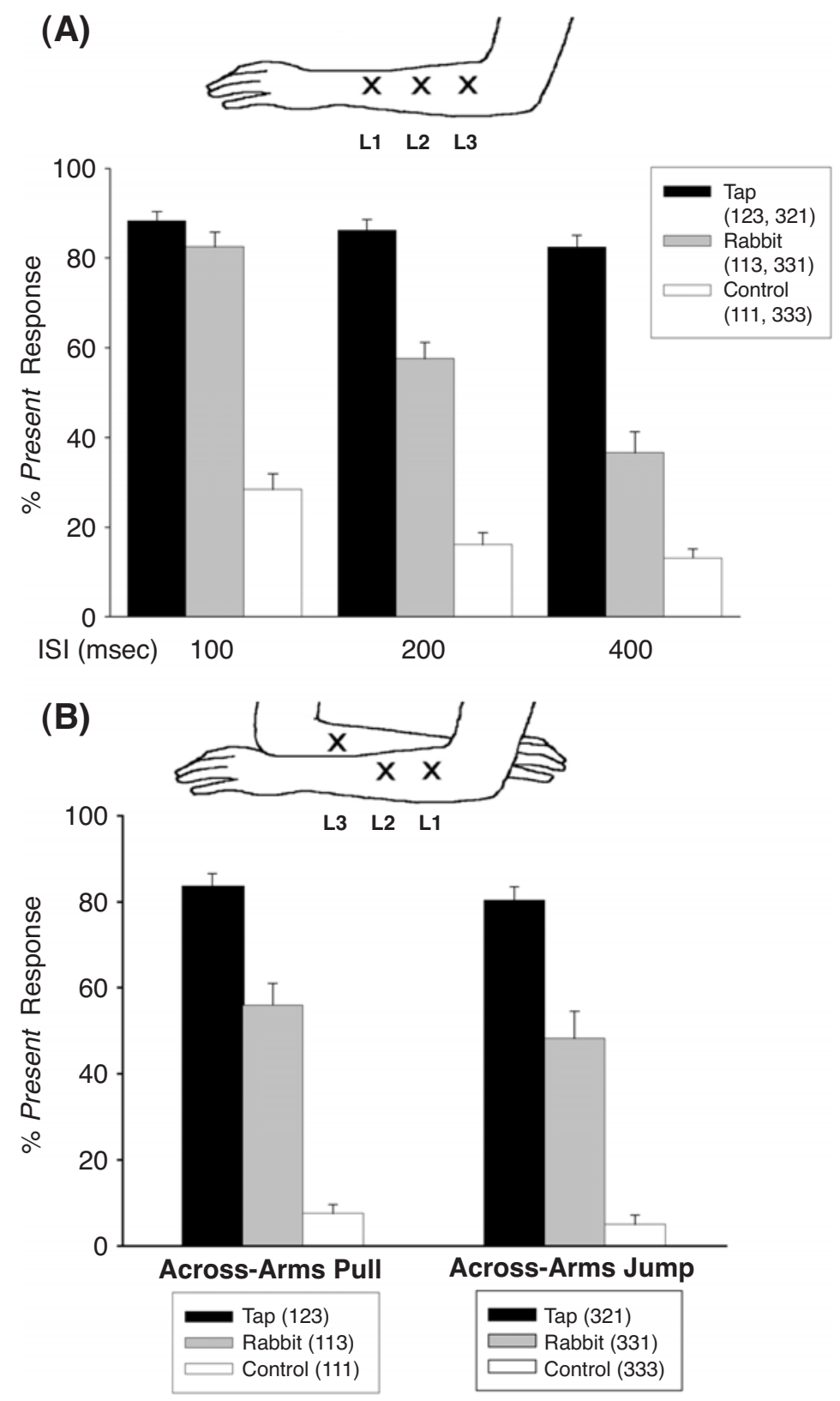

Figure 1. Stimulation locations and perceptual reports in the single-arm condition (A) and in the both-arms condition (B) of Experiment 1. Bar graphs show the mean percentage of trials where a tap was reported to be present at L2 (errors bars indicate $S E M$ ), displayed separately for tap, rabbit, and control trials. (A) Percentage of present responses in the single-arm condition for ISIs of $100 \mathrm{msec}, 200 \mathrm{msec}$, and $400 \mathrm{msec}$. (B) Percentage of present responses in the both-arms condition, shown separately for tap sequences starting at $\mathrm{L1}$ (with $\mathrm{T} 2$ delivered at $\mathrm{L1}$ on rabbit trials: across-arms pull, left) and for those starting at $\mathrm{L3}$ (with $\mathrm{T} 2$ delivered at $\mathrm{L} 3$ on rabbit trials: across-arms jump, right).

on one arm and L3 on the other (Figure 1B). If saltation was restricted to one side of the body, it should be completely eliminated when $\mathrm{T} 2$ and $\mathrm{T} 3$ were delivered to different arms. In the both-arms condition, the existence of a saltation illusion was assessed separately for trials start- ing at $\mathrm{L} 1$ and $\mathrm{L} 3$. In rabbit trials starting at L1, T2 was also delivered at L1, whereas $\mathrm{T} 3$ was presented at $\mathrm{L} 3$ on the other arm. Here, an illusory perception of T2 at L2 would demonstrate that perceived tap locations on one arm can be biased by a subsequent tactile event on the 
other arm ("across-arms pull" in Figure 1B). In rabbit trials starting at $\mathrm{L} 3, \mathrm{~T} 2$ was delivered at $\mathrm{L} 3$, followed by $\mathrm{T} 3$ at L1 on the other arm. Here, L3 (where T2 was presented) and L2 were located on opposite arms. Thus, perceived illusory shifts of T2 from L3 to L2 would indicate a mislocalization of $\mathrm{T} 2$ on the other arm ("across-arms jump" in Figure 1B), thereby suggesting that the cutaneous rabbit can hop across the body midline. Because the strength of saltation was expected to be reduced in the both-arms condition, T2-T3 ISIs were set to values close to the interval reported to be optimal for eliciting this illusion (50 and $100 \mathrm{msec}$ ). As in the single-arm condition, T1-T2 ISIs were always identical to T2-T3 ISIs.

\section{Method \\ Participants. Sixteen paid participants ( 9 female, 7 male; $19-32$ years of age) were tested.}

Stimuli, Apparatus, and Procedure. The participants received taps delivered via solenoids attached to three locations (L1, L2, L3) on the upper forearm. Solenoids drove a metal rod that contacted the arm for $6 \mathrm{msec}$. On each trial, three successive taps (T1, T2, T3) were delivered. The participants were instructed to direct their attention to the middle location (L2) and to report at the end of each trial, by pressing a left or right footpad, whether or not they had perceived a tap at this location. The assignment of present and absent responses to left and right footpads was balanced across participants. On tap trials, all three locations were successively stimulated $(\mathrm{L} 1 \rightarrow \mathrm{L} 2 \rightarrow \mathrm{L} 3 ; \mathrm{L} 3 \rightarrow \mathrm{L} 2 \rightarrow \mathrm{L} 1)$. On rabbit trials, $\mathrm{T} 1$ and $\mathrm{T} 2$ at one outer location were followed by T3 at the other outer location $(\mathrm{L} 1 \rightarrow \mathrm{L} 1 \rightarrow \mathrm{L} 3 ; \mathrm{L} 3 \rightarrow \mathrm{L} 3 \rightarrow \mathrm{L} 1)$. On control trials, all three stimuli were delivered successively at L1 or L3.

In the single-arm condition, all taps were delivered to the same arm. Stimulator locations corresponded to $33 \%, 50 \%$, and $67 \%$ of the elbow-wrist distance (Figure 1A). Eight participants received stimulation of the left arm, and the other 8 participants received rightarm stimulation. The ISI between successive taps was 100,200 , or $400 \mathrm{msec}$. Five successive blocks, consisting of 90 trials, were delivered. Each block contained 5 randomly intermingled trials for each combination of trial type (tap vs. rabbit vs. control), ISI (100 vs. 200 vs. $400 \mathrm{msec}$ ), and direction (sequences starting at L1 vs. L3).

In the both-arms condition, L1 and L2 were located on one arm (at 33\% and $50 \%$ of the elbow-wrist distance) and L3 on the other arm (at $33 \%$ of the elbow-wrist distance; Figure 1B). For 8 participants, L1 and L2 were on the left arm and L3 on the right arm, and this assignment was reversed for the other 8 participants. Intertap interval was 50 or $100 \mathrm{msec}$. Four successive blocks, consisting of 84 trials, were delivered. Each block contained 7 randomly intermingled trials for each combination of trial type, ISI, and direction.

Data Analysis. Trials without responses were excluded from analysis. Repeated measures analyses of variance (ANOVAs) were conducted on the percentage of present responses measured in the single-arm and both-arms conditions, separately for tap and rabbit trials (excluding control trials) and for rabbit and control trials (excluding tap trials). In the single-arm condition, analyses included the factors trial type (tap vs. rabbit; rabbit vs. control), direction, and ISI. For the both-arms condition, separate analyses were conducted for trial sequences starting at L1 (across-arms pull) and for trial sequences starting at L3 (across-arms jump), including the factors trial type and ISI. Additional paired $t$ tests were used to compare perceptual reports for different trial types. For all analyses, $\alpha$ level was .05 , and only statistically significant effects are reported.

\section{Results}

Single arm. Figure 1A shows percentages of present responses on tap, rabbit, and control trials in the single-arm condition. When tap and rabbit trials were analyzed together, an effect of trial type [tap vs. rabbit trials: $F(1,15)=$ $57.3, p<.001]$ showed that present responses were more frequent on tap trials than on rabbit trials. A trial type $\times$ ISI interaction $[F(2,30)=45.0, p<.001]$ indicated that the number of taps incorrectly reported at L2 on rabbit trials increased with decreasing ISIs. Paired $t$ tests revealed that taps at L2 were perceived less frequently on rabbit than on tap trials with 200- and 400-msec ISIs [ $t \mathrm{~s}(14)>8.0, p \mathrm{~s}<$ $.001]$. In contrast, for $100-\mathrm{msec}$ ISIs, the percentage of present responses on tap trials $(88 \%)$ and on rabbit trials (83\%) did not differ significantly, suggesting that real and illusory percepts were indiscriminable. When rabbit and control trials were analyzed together, an effect of trial type [rabbit vs. control trials: $F(1,15)=139.8, p<.001]$ demonstrated that present responses were much more frequent on rabbit than on control trials. Although a trial type $\times$ ISI interaction $[F(2,30)=23.4, p<.001]$ reflected the decrease of this saltation illusion with increasing ISI, paired $t$ tests revealed that participants reported illusory taps at L2 more frequently on rabbit than on control trials for all three ISIs $[t \mathrm{~s}(15)>4.9, p \mathrm{~s}<.001]$.

Both arms. Figure 1B shows percentages of present responses of tap, rabbit, and control trials in the botharms condition, displayed separately for tap sequences starting at L1 (with illusory displacements of T2 from L1 to L2 on the same arm representing an across-arms pull; left side) and for sequences starting at L3 (with illusory displacements of T2 from L3 to L2 on the opposite arm representing an across-arms jump; right side). Because no main effects of ISI or interactions including this factor were present, the data shown in Figure 1B are averaged across both ISIs (50 and $100 \mathrm{msec}$ ). When tap and rabbit trials were analyzed together, main effects of trial type (tap vs. rabbit trials) were obtained for both movement directions $[F(1,15)=19.3$ and 15.2, $p$ s $<$ .001 , respectively], reflecting the fact that present responses were more frequent on tap than on rabbit trials. When rabbit and control trials were analyzed together, main effects of trial type (rabbit vs. control trials) were again obtained for both movement directions $[F(1,15)=$ 114.2 and $63.9, p s<.001$, respectively]. This finding that present responses were much more likely on rabbit than on control trials indicates the presence of a saltation illusion in the both-arms condition and suggests that this illusion was elicited regardless of whether T2 was presented at L1, and therefore could be mislocated on the same arm, or was presented at L3 and thus could be mislocated on the opposite arm (Figure 1B). Paired $t$ tests confirmed that present responses were more frequent on rabbit than on control trials for sequences starting at L1 as well as at $\mathrm{L} 3[t \mathrm{~s}(15)>8.0, p \mathrm{~s}<.001]$.

\section{Discussion}

In Experiment 1, we used a newly developed procedure to objectively measure the strength of the cutaneous saltation illusion, producing several noteworthy findings. The results from the single-arm condition showed that the strength of the saltation illusion decreased with 
increasing T2-T3 ISIs. For 100-msec intervals, illusory taps at L2 on rabbit trials were reported as frequently as were real taps on tap trials, thereby suggesting that real and illusory tap locations were perceptually very similar. With longer T2-T3 ISIs (200 and $400 \mathrm{msec}$ ), participants were less likely to report the presence of a tap at L2 on rabbit trials than on tap trials. In line with earlier experiments using subjective location judgments (Geldard, 1975, 1982), this result shows that the strength of the saltation illusion is sensitive to the T2-T3 ISI. Furthermore, illusory taps at L2 were generally reported much more frequently on rabbit trials than on control trials in the single-arm condition. Since these trials were designed to be equivalent with respect to spatial biases produced by expectation and attention, this finding strongly suggests that tactile saltation cannot be explained solely in terms of an attentional bias (Kilgard \& Merzenich, 1995).

The results obtained in the both-arms condition provide evidence that illusory displacements of stimuli delivered to one side of the body can be elicited by tactile stimuli subsequently delivered to the opposite side. Illusory taps at L 2 were reported on about $50 \%$ of all rabbit trials (and on less than $10 \%$ of control trials). The fact that participants were more likely to report the presence of a tap at L2 on rabbit trials than on control trials when stimulus sequences started at L1 suggests that perceived tap locations on one arm are systematically shifted toward a subsequent tap on the other arm ("across-arms pull" in Figure 1B). Similar findings were also obtained for sequences starting at L3, suggesting that taps were frequently mislocalized on the wrong arm when this arm was subsequently stimulated ("across-arms jump" in Figure 1B). This result seriously challenges the hypothesis that the cutaneous rabbit is unable to cross the body midline. Overall, the perceptual reports obtained in the both-arms condition indicate the presence of a strong biasing effect exerted by tactile stimuli presented to one arm on location judgments in response to stimuli previously delivered to the other arm.

Before these interpretations can be accepted, potential shortcomings of the experimental procedure used in Experiment 1 need to be considered. In the single-arm condition, taps were more likely to be reported at L2 on rabbit trials than on control trials even when the T2-T3 ISI was $400 \mathrm{msec}$, which is clearly outside the temporal range for which cutaneous saltation was observed in earlier studies (Cholewiak, 1976; Geldard, 1975). This unexpected finding suggests that perceptual reports on rabbit trials may have been affected by factors other than cutaneous saltation. One possibly problematic aspect of the procedure employed in Experiment 1 was that stimulus sequences were stationary on control trials (where all three taps were presented at the same location), whereas on rabbit and on tap trials, successive taps were delivered to different locations. It is possible that participants may have been biased to report a tap at L2 whenever a sequence was not stationary, especially when ISIs were short, and that this response bias caused different perceptual reports on rabbit and control trials. The fact that
ISIs were randomized within blocks may even have enhanced the differential salience of stationary versus moving stimulus sequences, thereby potentially exacerbating possible response bias effects. In order to interpret the findings of Experiment 1 as evidence for cutaneous saltation, the potential impact of response bias needs to be controlled. Experiment 2 was conducted to address this issue.

Another possible problem of Experiment 1 is related to the fact that the T1-T2 and T2-T3 ISI were identical. This procedure differs somewhat from the procedure used in earlier investigations of cutaneous saltation (e.g., Geldard, 1982), where the T1-T2 ISI was typically much longer $(800 \mathrm{msec})$ than the $\mathrm{T} 2-\mathrm{T} 3$ interval. As described earlier, identical T1-T2 and T2-T3 ISIs were chosen in order to neutralize any biasing influence of the tau effect (Helson \& King, 1931) on perceptual reports. However, presenting successive tactile stimuli with ISIs of $100 \mathrm{msec}$ or less can give rise to apparent motion perceptions (tactual phi; Sherrick \& Rogers, 1966), which could have contributed to the effects observed in Experiment 1. To reduce any possible impact of induced apparent motion and to test our new measure of saltation with temporal stimulation procedures identical to those used in earlier studies, Experiment 2 featured T1-T2 intervals of $800 \mathrm{msec}$ in all trials.

\section{EXPERIMENT 2}

The aim of Experiment 2 was to confirm the results observed in Experiment 1, while at the same time controlling for the possible impact of response bias effects induced by the presence or absence of stimulus movement. In addition, Experiment 2 employed T1-T2 intervals identical to those used in classical investigations of cutaneous saltation (e.g., Geldard, 1975, 1982). The procedures were identical to those of Experiment 1, with the following exceptions. First, the T1-T2 ISI was now $800 \mathrm{msec}$ in all trials. The T2-T3 ISI was set to $100 \mathrm{msec}$ (where the saltation illusion should be strong) or $300 \mathrm{msec}$ (where this illusion should be weak or absent), for both the singlearm and both-arms conditions. Short and long T2-T3 ISIs were now presented in separate blocks, rather than randomized within blocks, as in Experiment 1. Finally, and most important, an additional control condition was introduced. On motion control trials, T1 at one of the outer locations was followed by $\mathrm{T} 2$ and $\mathrm{T} 3$ at the other outer location $(\mathrm{L} 1 \rightarrow \mathrm{L} 3 \rightarrow \mathrm{L} 3 ; \mathrm{L} 3 \rightarrow \mathrm{L} 1 \rightarrow \mathrm{L} 1)$. These trials were similar to rabbit and tap trials in that tactile sequences were not stationary, but involved the successive stimulation of different locations. ${ }^{2}$ If the perceptual reports observed for rabbit trials in Experiment 1 were affected by a differential response bias induced by stationary versus moving stimulation sequences, this bias should also be present on motion control trials. If response bias was solely responsible for the difference between perceptual reports obtained on rabbit and control trials in Experiment 1, taps at L2 should now be reported equally often on rabbit trials and on motion control trials. In contrast, genuine cutaneous saltation effects will be reflected by 
more frequent present responses on rabbit trials than on motion control trials.

\section{Method}

Participants. Ten participants ( 6 female, 4 male; $21-42$ years of age) were tested. None had participated in Experiment 1.

Stimuli, Apparatus, Procedure, and Data Analysis. The locations of tactile stimulators, arm postures, and participants' task were the same as in Experiment 1. The T1-T2 ISI was now always $800 \mathrm{msec}$, and the T2-T3 ISI was set to 100 or $300 \mathrm{msec}$, in different blocks. In addition to the three trial types (tap, rabbit, control) included in Experiment 1, motion control trials were included, where $\mathrm{T} 1$ at one outer location was followed by T2 and T3, which were both presented at the opposite outer location $(\mathrm{L} 1 \rightarrow \mathrm{L} 3 \rightarrow \mathrm{L} 3$; $\mathrm{L} 3 \rightarrow \mathrm{L} 1 \rightarrow \mathrm{L} 1)$. The control condition already included in Experiment $1(\mathrm{~L} 1 \rightarrow \mathrm{L} 1 \rightarrow \mathrm{L} 1 ; \mathrm{L} 3 \rightarrow \mathrm{L} 3 \rightarrow \mathrm{L} 3)$ is now referred to as static control.

The experiment consisted of 12 blocks, and each block contained 40 trials. Six successive blocks were delivered for the single-arm and both-arms conditions, respectively. Each block contained 5 randomly intermingled trials for each combination of trial type (tap vs. rabbit vs. static control vs. motion control) and direction. In three successive blocks, the T2-T3 ISI was $100 \mathrm{msec}$, and in the other three successive blocks, the ISI was $300 \mathrm{msec}$. In all other respects, the procedures were identical to those of Experiment 1. Data analyses were analogous to Experiment 1, except that additional repeated measures ANOVAs were conducted to compare perceptual reports for rabbit and for motion control trials.

\section{Results}

Single arm. Figure 2 shows the percentage of present responses on tap, rabbit, static control, and motion con- trol trials in the single-arm condition for T2-T3 ISIs of $100 \mathrm{msec}$ (left) and $300 \mathrm{msec}$ (right). When tap and rabbit trials were analyzed together, an effect of trial type [tap vs. rabbit trials: $F(1,9)=9.3, p<.02$ ] showed that present responses were more frequent on tap than on rabbit trials. A trial type $\times$ ISI interaction $[F(1,9)=30.5$, $p<.001]$ indicated that incorrectly reported taps at L2 on rabbit trials were more frequent for the shorter ISI. Paired $t$ tests revealed that taps at $\mathrm{L} 2$ were perceived less frequently on rabbit than on tap trials at ISIs of $300 \mathrm{msec}$ $[t(9)=4.6, p<.01]$. In contrast, for 100 -msec ISIs, the percentage of present responses on tap trials $(81 \%)$ and rabbit trials $(82 \%)$ did not differ significantly, thus again suggesting that real and illusory percepts may be phenomenologically indistinguishable when $\mathrm{T} 2$ and $\mathrm{T} 3$ are separated by $100 \mathrm{msec}$. When rabbit and static control trials were analyzed together, an effect of trial type [rabbit vs. static control trials: $F(1,9)=95.5, p<.001]$ demonstrated that present responses were more frequent on rabbit than on static control trials. Although a trial type $\times$ ISI interaction $[F(1,9)=17.2, p<.01]$ indicated that this difference was larger with ISIs of $100 \mathrm{msec}$, paired $t$ tests revealed that participants reported illusory taps at L2 more frequently on rabbit trials than on static control trials for both ISIs [ts $(9)>4.5, p \mathrm{~s}<.001]$. Importantly, when rabbit and motion control trials were analyzed together, an effect of trial type [rabbit vs. motion control trials: $F(1,9)=8.9, p<.02]$ demonstrated that present responses were more frequent on rabbit trials
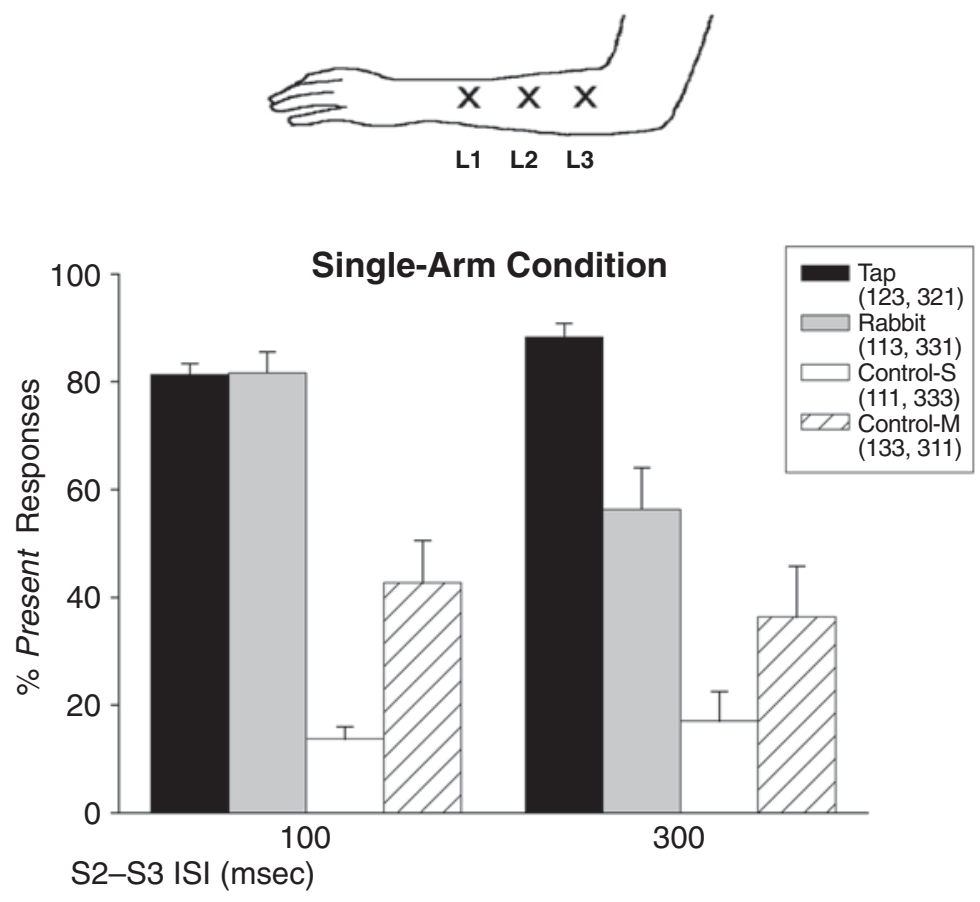

Figure 2. Stimulation locations and perceptual reports in the single-arm condition of Experiment 2. Bar graphs show the mean percentage of trials where a tap was reported to be present at L2 (errors bars indicate $S E M$ ), displayed separately for tap, rabbit, static control (control-S), and motion control (control-M) trials and for T2-T3 ISIs of $100 \mathrm{msec}$ (left) and $300 \mathrm{msec}$ (right). 
than on motion control trials. Again, a trial type $\times$ ISI interaction $[F(1,9)=8.2, p<.02]$ reflected the decrease of this difference with increasing ISI. Paired $t$ tests revealed that the difference in the number of illusory taps reported at L2 on rabbit and motion control trials was not significant for 300-msec ISIs. In contrast, for ISIs of $100 \mathrm{msec}$, participants reported illusory taps at L2 significantly more often on rabbit trials than on motion control trials $[t(9)=3.9, p<.01]$.

Both arms. Figure 3 shows percentages of present responses of tap, rabbit, static control, and motion control trials in the both-arms condition for blocks where the T2-T3 ISIs were $100 \mathrm{msec}$ (top panel) and $300 \mathrm{msec}$ (bottom panel). Results are shown separately for trials where the tap sequence started at L1 (with any acrossarms pull resulting in illusory displacements of $\mathrm{T} 2$ from L1 to L2 on the same arm; Figure 3, left side) and for sequences starting at L3 (with any across-arms jump resulting in perceived displacements of T2 across arms from L3 to L2; Figure 3, right side). When tap and rabbit trials were analyzed together, significant effects of trial type (tap vs. rabbit trials) were present for both movement directions $[F(1,9)=31.4$ and 21.9, $p$ s $<.001$, respectively], demonstrating that present responses were more frequent on tap than on rabbit trials. A trial type $\times$ ISI interaction was significant for trials starting at L1 $[F(1,9)=7.6, p<.03]$, indicating that taps were more likely to be incorrectly reported at L2 on rabbit trials when ISI was $100 \mathrm{msec}$. When rabbit and static control trials were analyzed together, significant effects of trial type (rabbit vs. static control trials) were again present for both movement directions $[F(1,9)=13.9$ and 21.7, $p \mathrm{~s}<.005$, respectively]. As in Experiment 1, present responses were more frequent on rabbit trials than on static control trials for both movement directions, thus apparently again suggesting the presence of an across-arms pull effect as well as an across-arms jump.

However, when rabbit trials and the newly introduced motion control trials were analyzed together, a different picture emerged. Importantly, no significant main effect of trial type (rabbit vs. motion control trials) or trial type $\times$ ISI interaction was found for trials starting at L3, thus demonstrating that on these trials, taps were no more likely to be incorrectly reported at $\mathrm{L} 2$ on rabbit trials than on motion control trials (see Figure 3, right: across-arms jump). In contrast, a significant effect of trial type (rabbit vs. motion control trials) was present for trials starting at $\mathrm{L} 1[F(1,9)=9.8, p<.02]$, since present responses were more frequent on rabbit trials than on motion control trials (see Figure 3, left: acrossarms pull). Although the trial type $\times$ ISI interaction was not significant, this difference in perceptual judgments between rabbit and motion control trials tended to be much more pronounced with ISIs of $100 \mathrm{msec}$. Follow-up paired $t$ tests conducted for trials starting at $\mathrm{L} 1$ confirmed that for the 100-msec ISI, the percentage of present responses on rabbit trials $(68 \%)$ and on motion control trials (38\%) differed significantly $[t(9)=2.4, p<.04]$, thereby demonstrating the presence of a reliable across-arms pull effect. In contrast, this difference failed to reach significance for the $300-\mathrm{msec}$ ISI ( $36 \%$ and $20 \%$ present responses on rabbit and motion control trials, respectively).

\section{Discussion}

The results observed in the single-arm condition of Experiment 2 were very similar to those previously found in Experiment 1. As in this first experiment, perceptual reports were identical for rabbit trials and tap trials when the T2-T3 ISI was $100 \mathrm{msec}$, thereby suggesting again that these two types of trials are perceptually very similar, or even indistinguishable. The latter claim was further investigated in Experiment 3. An important difference in Experiment 1 was that the T1-T2 ISI was now $800 \mathrm{msec}$ (as in earlier investigations of saltation; e.g., Geldard, 1975, 1982). Although it has previously been claimed that T1-T2 ISIs need to be at least $300 \mathrm{msec}$ to produce a reliable saltation illusion (Geldard, 1982), the present results do not support this view. The fact that perceptual reports for rabbit and tap trials were statistically equivalent in Experiment 1 for T1-T2 ISIs of $100 \mathrm{msec}$, as well as in Experiment 2 for ISIs of $800 \mathrm{msec}$, suggests that variations in the T1-T2 interval do not affect the strength of the saltation illusion.

As in Experiment 1, present responses were more frequent on rabbit trials than on static control trials in the single-arm condition, both when the T2-T3 ISI was $100 \mathrm{msec}$, but also for ISIs of $300 \mathrm{msec}$. Crucially, the newly introduced motion control condition made it possible to dissociate effects of response bias triggered by the difference between static and moving stimulation sequences on rabbit trials from genuine cutaneous saltation effects. As shown in Figure 2, participants were more likely to report the presence of a tap at L2 on motion control trials (where successive taps were delivered to different locations; $39.5 \%$ present responses) than on static control trials (where all taps were presented at the same location; $15.3 \%$ present responses). This difference strongly suggests that response bias is likely to be responsible for at least some of the present responses on rabbit trials. However, and importantly, when the T2-T3 ISI was $100 \mathrm{msec}$, taps were significantly more often reported at L2 on rabbit trials ( $82 \%$ ) than on motion control trials (43\%). This result demonstrates the presence of a genuine saltation illusion, above and beyond any biasing effects of perceived motion. In contrast, when the T2-T3 ISI was longer (300 msec), no significant differences in perceptual reports between rabbit and motion control trials were obtained. Thus, for this longer interval, differences in perceptual reports between rabbit and static control trials are primarily caused by response bias, and not by cutaneous saltation.

The results obtained in the both-arms condition of Experiment 2 for tap, rabbit, and static control trials when the T2-T3 ISI was $100 \mathrm{msec}$ were again very similar to those obtained in Experiment 1 (compare Figure 1B and Figure 3A). Taps were less likely to be reported at L2 on rabbit trials than on tap trials, but these reports were considerably more frequent on rabbit trials than on static 

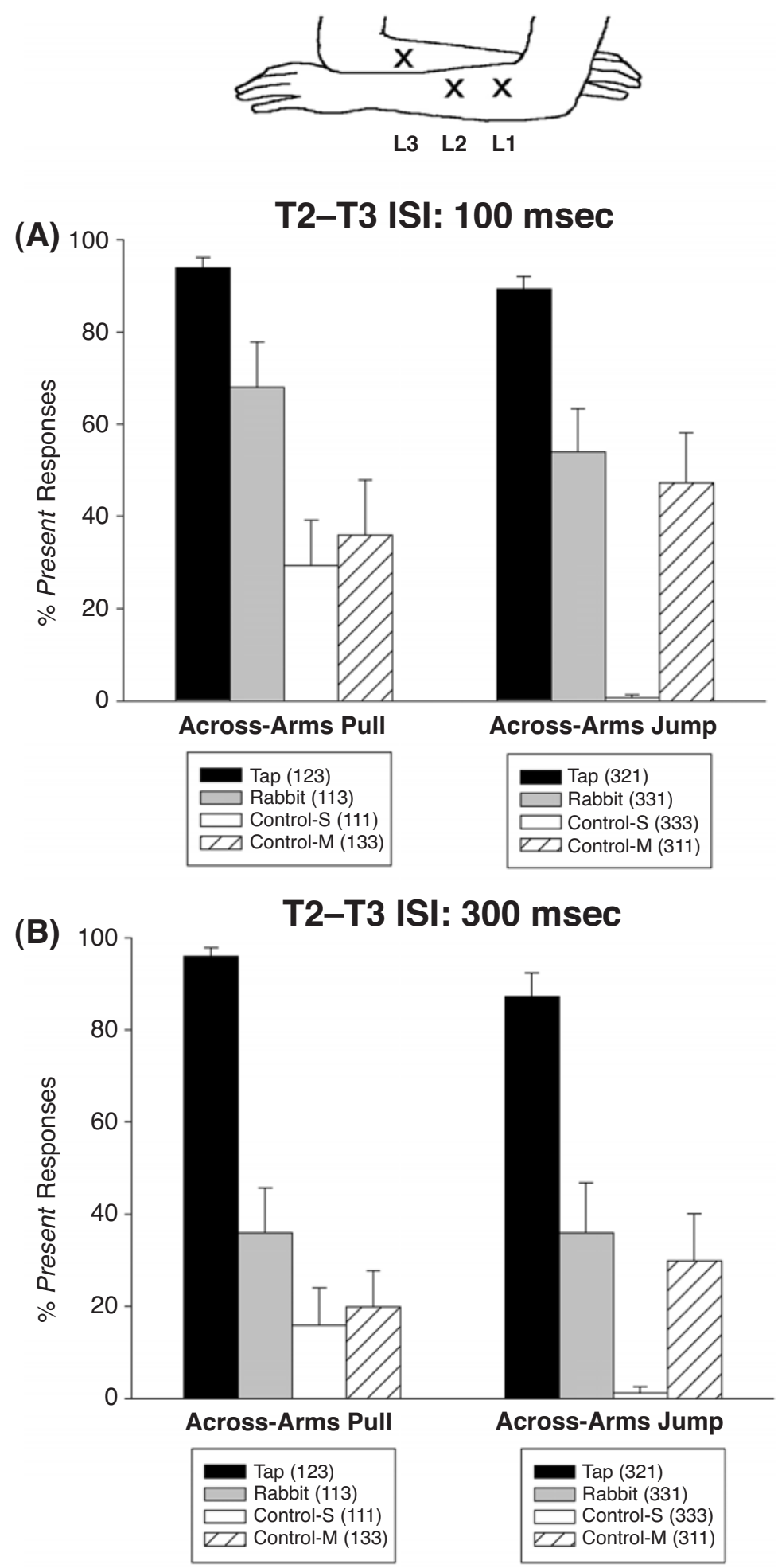

Figure 3. Stimulation locations and perceptual reports in the both-arms condition of Experiment 2. Bar graphs show the mean percentage of trials where a tap was reported to be present at $L 2$ (errors bars indicate $S E M$ ), displayed separately for tap, rabbit, static control (control-S), and motion control (control-M) trials. Data are shown separately for tap sequences starting at L1 (with T2 delivered at L1 on rabbit trials: "across-arms pull"; left) and for those starting at L3 (with T2 delivered at L3 on rabbit trials: "across-arms jump"; right). (A) Percentage of present responses for T2-T3 ISI of $100 \mathrm{msec}$. (B) Percentage of present responses for T2-T3 ISI of 300 msec. 
control trials. We tentatively interpreted analogous findings in Experiment 1 as evidence for the ability of the cutaneous rabbit to hop across arms. However, the results obtained in Experiment 2 for motion control trials qualify this interpretation in an important way. For trials starting at L3 (trials where cutaneous saltation would imply the mislocalization of a tactile event on the opposite arm; Figure 3: across-arms jump), perceptual reports on rabbit trials and motion control trials did not differ significantly. This finding indicates that the differences observed in both experiments between rabbit and static control trials starting at L3 can be fully accounted for by response bias triggered by perceived stimulus movement and thus should not be regarded as evidence that the cutaneous rabbit can jump across arms.

However, a different pattern of results was obtained in the both-arms condition for trials starting at L1 (trials where cutaneous saltation would indicate that tactile events delivered to one arm affect the localization of an earlier tactile event on the opposite arm; Figure 3: acrossarms pull). When the T2-T3 ISI was $100 \mathrm{msec}$, participants were significantly more likely to report the presence of a tap at L2 on rabbit trials than on motion control trials. Since perceptual reports on these trials should have been equally affected by response bias induced by stimulus motion, this difference appears to reflect the presence of a genuine across-arms localization bias. This finding supports our tentative conclusion regarding the findings in Experiment 1 that sensory saltation effects on one side of the body midline can be triggered by a subsequent tactile stimulus presented to the opposite side.

\section{EXPERIMENT 3}

In the single-arm condition of Experiments 1 and 2, participants were equally likely to report the presence of a tap at L2 on rabbit trials and on tap trials when the T2-T3 ISI was $100 \mathrm{msec}$. This fact was tentatively interpreted as indicating that illusory tap locations on rabbit trials were perceptually similar or perhaps even identical to real taps at L2 on tap trials. However, the presence of statistically equivalent perceptual reports on tap and on rabbit trials should not be interpreted as conclusive evidence for the strong claim that observers' percepts on these two types of trials were phenomenologically identical. It is entirely conceivable that perceived tap locations at L2 were generally more salient on tap trials when L2 was in fact stimulated than on rabbit trials. ${ }^{3}$

In Experiment 3, we employed a two-alternative forced choice procedure in order to investigate directly whether or not perceived tap locations on rabbit trials and on tap trials are perceptually indistinguishable. On every trial, two tactile stimulation sequences (a tap sequence including stimulation of L2 and a rabbit sequence without L2 stimulation, as defined in Experiments 1 and 2) were presented successively on the same arm and in random order across trials. Participants had to decide at the end of each trial whether the first or the second stimulus sequence contained a tap at L2. If their percepts on rabbit and on tap sequences were phenomenologically identical, forced choice discrimination performance should be at chance level. In contrast, if perceived tap locations at L2 were more salient during tap sequences than during rabbit sequences, observers should be able to use this phenomenological difference to reliably identify the stimulation sequence that contained a real stimulation of $\mathrm{L} 2$.

In the first part of Experiment 3, each trial contained one tap and one rabbit sequence, and observers were asked at the end of each block to rate their confidence with respect to their ability to identify the sequence containing a tap at $\mathrm{L} 2$. In the second part, trials containing tap and rabbit sequences were randomly intermixed with two-alternative forced choice trials containing tap and motion control sequences (as defined in Experiment 2). Since no saltation illusion should be elicited during motion control sequences, these trials were included to obtain a baseline measure of participants' two-alternative forced choice accuracy. In addition, their presence might provide observers with a clearly discriminable perceptual difference, which could be used to anchor their presumably more subtle perceptual judgments on trials including tap and rabbit sequences.

\section{Method}

Participants. Ten participants (4 female, 6 male; $24-35$ years of age) were tested. None had participated in Experiments 1 or 2.

Stimuli, Apparatus, and Procedure. The locations of the tactile stimulators were the same as in Experiments 1 and 2. All taps were delivered to the same arm (the left arm for 5 participants, the right arm for the other 5 participants). As in Experiment 2, the T1-T2 ISI was $800 \mathrm{msec}$, and the T2-T3 ISI was $100 \mathrm{msec}$. On each trial, two tactile stimulus sequences were presented successively, separated by an empty interval of 1,000 msec. Both sequences always started at the same location (L1 or L3), and one of them was a tap sequence $(\mathrm{L} 1 \rightarrow \mathrm{L} 2 \rightarrow \mathrm{L} 3 ; \mathrm{L} 3 \rightarrow \mathrm{L} 2 \rightarrow \mathrm{L} 1)$, whereas the other was either a rabbit sequence $(\mathrm{L} 1 \rightarrow \mathrm{L} 1 \rightarrow \mathrm{L} 3 ; \mathrm{L} 3 \rightarrow \mathrm{L} 3 \rightarrow \mathrm{L} 1)$ or a motion control sequence, as defined in Experiment $2(\mathrm{~L} 1 \rightarrow \mathrm{L} 3 \rightarrow \mathrm{L} 3 ; \mathrm{L} 3 \rightarrow \mathrm{L} 1 \rightarrow \mathrm{L} 1)$. The two possible serial positions of a tap sequence within a trial (first vs. second) were equiprobable and randomized across trials. The participants were instructed to report at the end of each trial which of the two stimulus sequences included a tap at the middle location (L2) by pressing a left or right footpad. Five participants used the left footpad to signal the presence of a tap at L2 in the first sequence and the right footpad for a tap at L2 in the second sequence; these response assignments were reversed for the other 5 participants.

The experiment consisted of two parts. In the first part, tap sequences were always paired with rabbit sequences. Three blocks were run, each consisting of 12 trials. At the end of each block, the participants rated their overall confidence with respect to the accuracy of their two-alternative forced choice judgments on a 7-point scale $(1=I$ was guessing all the time, $4=I$ was guessing on half of all trials, and $7=$ I am confident that I was always correct $)$. The second part of the experiment consisted of three blocks (48 trials per block). Tap sequences were paired with rabbit sequences on 32 trials per block and with motion control sequences on 16 trials per block. The order in which these two types of trials were presented was completely randomized. No confidence ratings were required.

\section{Results and Discussion}

In the first part of Experiment 3, where tap and rabbit sequences were always paired, participants correctly identified which of the two successively presented se- 
quences contained a tap at L2 on 59\% of all trials. This detection performance did not differ significantly from chance [ $50 \%$ correct; $t(9)=1.0, p=.32$ ]. Observers' mean confidence rating was 4.0 , indicating that on average, they judged themselves to have been able to correctly identify the tap sequence on $50 \%$ of all trials. However, confidence was not predictive of actual discrimination performance, because participants' confidence ratings after each experimental block and their actual detection accuracy in these blocks were completely uncorrelated $(r=.04)$.

In the second part of the experiment, where tap sequences were paired with rabbit sequences on two thirds of all trials and with motion control sequences on the remaining trials, forced choice discriminative judgments were significantly more accurate when trials contained a tap-motion control pairing than when trials contained a tap-rabbit pairing $[t(9)=4.6, p<.01]$. Participants correctly identified the tap sequence on $85 \%$ of all trials when it was paired with a motion control sequence, which was clearly above chance $[t(9)=13.4, p<.001]$. In contrast, tap detection accuracy was again only $59 \%$ on trials where tap and rabbit sequences were paired, which was not different from chance performance $[t(9)=1.1$, $p=.20]$.

Overall, the two-alternative forced choice results from Experiment 3 provide support for the hypothesis that observers' percepts on rabbit trials and on tap trials are phenomenologically very similar. Although there was a tendency in favor of observers choosing the tap sequence, overall discrimination performance across observers did not differ significantly from chance, regardless of whether tap-rabbit sequence pairings were presented in isolation or randomly intermingled with tap-motion control pairs. Overall, there was substantial variability in observers' discriminative judgments in response to tap-rabbit sequence pairings, suggesting considerable interindividual differences in the strength of the saltation illustration. In fact, several observers (4 in the first part and 2 in the second part of Experiment 3 ) were actually more likely to pick the rabbit sequence than the tap sequence. However, all 10 observers performed better (and consistently well above chance) when distinguishing between tap and motion control sequences as opposed to tap-rabbit sequences. If perceived tap locations at L2 had been clearly more salient during tap sequences than during rabbit sequences, observers could have based their discriminative judgments on this perceptual difference and therefore should have been able to choose tap sequences in the majority of trials. The fact that overall discrimination performance was at chance level provides additional evidence for the strength of the saltation illusion by objectively demonstrating observers' difficulty of discriminating between real and illusory tap locations.

\section{GENERAL DISCUSSION}

The aim of the present experiments was to introduce and evaluate a new objective procedure to measure the strength of the cutaneous rabbit illusion and to use this measure to investigate and clarify controversies with respect to the nature of this illusion. On each trial, a sequence of three taps (T1, T2, T3) was presented to three possible locations (L1, L2, L3) on the left or right forearm, and participants had to report whether or not a tap was delivered to the middle location (L2). Perceptual reports on trials where L2 was in fact stimulated (tap trials) were compared with perceptual reports on trials where two successive taps at one of the outer locations were followed by one tap at the other outer location (this was expected to produce an illusory perception of T2 at L2; rabbit trials). Identical perceptual reports on these two types of trials would suggest that real and illusory taps at L2 are perceptually equivalent, and this was indeed found in Experiments 1 and 2 when all stimuli were delivered to the same arm, and the T2-T3 ISI was $100 \mathrm{msec}$. Under these conditions, participants were just as likely to report a tap at L2 in rabbit trials as in tap trials. We used a two-alternative forced choice procedure in Experiment 3 to demonstrate that observers were generally unable to distinguish between real and illusory tap locations, thereby suggesting that the percepts elicited on tap and rabbit trials were phenomenologically very similar, and for most observers possibly even equivalent. Taken together, these findings emphatically underline the strength of the cutaneous saltation illusion.

The finding that taps were more likely to be reported at L2 on rabbit trials than on control trials (static control in Experiments 1 and 2, motion control in Experiment 2) rules out the hypothesis that saltation can be explained exclusively in terms of biases produced by anticipation and selective attention (Kilgard \& Merzenich, 1995). The participants were explicitly instructed to attend to L2 in order to detect the presence or absence of a tap at that location. With attention continuously focused at L2, perceived $\mathrm{T} 2$ locations should have been affected by equivalent spatial biases in rabbit trials and in control trials, resulting in similar perceptual reports. The fact that perceptual judgments were in fact markedly different strongly suggests that cutaneous saltation is not just an artifact of spatial attention. ${ }^{4}$

In line with earlier findings that sensory saltation critically depends on the interval separating T2 and T3 (Geldard, 1975, 1982), the percentage of taps incorrectly reported at L2 on rabbit trials decreased as this interval was increased. The results obtained in the single-arm condition of Experiment 2 suggest that cutaneous saltation is virtually absent in 300 -msec ISIs, and present responses on rabbit trials reflect a response bias caused by perceived stimulus movement. Response bias effects are also likely to be responsible for the substantial number of present responses for rabbit trials in the single-arm condition of Experiment 1 when ISIs were $400 \mathrm{msec}$ (and therefore well outside the range where the saltation illusion is normally elicited).

To reevaluate the claim that the cutaneous rabbit is confined to one side of the body and never crosses the body midline (Geldard, 1982; Geldard \& Sherrick, 1983), 
successive stimuli were delivered to different arms in the both-arms condition. Here, the presence of the saltation illusion was evaluated independently for trials starting at L3 (where present responses on rabbit trials would indicate the illusory localization of taps on the wrong arm; across-arms jump) and for trials starting at L1 (where present responses on rabbit trials would indicate a mislocalization of taps on one arm caused by a subsequent tap on the opposite arm; across-arms pull). The results obtained in Experiment 1 suggested the presence of an across-arms pull as well as an across-arms jump, but Experiment 2 indicated that the apparent across-arms jump can be fully accounted for by response bias alone. Thus, the findings of the present study are in line with earlier observations by Geldard and colleagues that sensory saltation does not produce illusory shifts of tactile events across the body midline. In other words, the cutaneous rabbit remained unwilling to jump across arms. However, a reliable across-arms pull remained present in Experiment 2 above and beyond any effects of response bias triggered by perceived motion. This finding that the location of a tactile event delivered to one arm is shifted toward a subsequent tactile event presented to the other arm is important, since it demonstrates that this illusion can occur even when successive tactile events are initially represented in different hemispheres. This observation appears to rule out the primary somatosensory cortex (S1) as the basis of sensory saltation, since S1 exclusively represents the contralateral body side and has few commissural connections to the opposite hemisphere (Powell, 1977). Unlike S1, higher order somatosensory areas (secondary and posterior parietal somatosensory cortex) contain a large number of neurons with bilateral receptive fields (Iwamura, Iriki, \& Tanaka, 1994). These areas may have mediated the tactile localization bias on one arm produced by a subsequent tactile event on the other arm.

In summary, the present experiments have demonstrated the utility of a new objective measure of cutaneous saltation and have challenged previous theoretical accounts of this illusion. On the methodological level, the similarity of perceptual reports in Experiments 1 and 2 observed despite differences in the T1-T2 ISI, and despite the fact that ISI conditions were blocked in Experiment 2 and randomized within blocks in Experiment 1, underlines the reliability of perceptual reports as measures of cutaneous saltation. With respect to the validity of this measure, Experiment 2 revealed that response bias effects related to the difference between stationary and moving tactile stimulation sequences represent a potential confounding factor. This factor needs to be controlled in order to be able to interpret perceptual reports as valid indicators of the saltation illusion.

On the theoretical level, the present findings, and especially the results of Experiment 3, suggest that cutaneous saltation is sufficiently powerful to produce illusory percepts that are highly similar to, or perhaps even phenomenologically indistinguishable from, veridical tactile sensations. Although the saltation illusion clearly cannot be fully explained in terms of top-down factors such as attention and expectation (Kilgard \& Merzenich, 1995), the fact that a tap on one arm can affect the localization of a previous tap on the other arm is also inconsistent with the view that tactile saltation originates at early somatosensory processing stages (Geldard, 1975, 1982) and suggests that this illusion is either generated in higher order somatosensory areas or is a postperceptual phenomenon.

\section{REFERENCES}

Cholewiak, R. W. (1976). Satiation in cutaneous saltation. Sensory Processes, 1, 163-175.

Cholewiak, R. W. (1999). The perception of tactile distance: Influences of body site, space, and time. Perception, 28, 851-875.

Cholewiak, R. W., \& Collins, A. A. (2000). The generation of vibrotactile patterns on a linear array: Influences of body site, time, and presentation mode. Perception \& Psychophysics, 62, 1220-1235.

DennetT, D. C. (1991). Consciousness explained. Boston: Little, Brown. GELDARD, F. A. (1975). Sensory saltation: Metastability in the perceptual world. Hillsdale, NJ: Erlbaum.

Geldard, F. A. (1982). Saltation in somesthesis. Psychological Bulletin, 92, 136-175.

Geldard, F. A., \& Sherrick, C. E. (1972). The cutaneous "rabbit": A perceptual illusion. Science, 178, 178-179.

Geldard, F. A., \& Sherrick, C. E. (1983). The cutaneous saltatory area and its presumed neural basis. Perception \& Psychophysics, 33, 299-304.

Helson, H., \& King, S. M. (1931). The tau effect: An example of psychological relativity. Journal of Experimental Psychology, 14, 202217.

IwAMURA, Y., IRIKI, A., \& TANAKA, M. (1994). Bilateral hand representation in the postcentral somatosensory cortex. Nature, 369, 554556.

Kilgard, M. P., \& Merzenich, M. M. (1995). Anticipated stimuli across skin. Nature, 373, 663 .

Powell, T. P. S. (1977). The somatic sensory cortex. British Medical Bulletin, 33, 129-136.

Sherrick, C. E., \& Rogers, R. (1966). Apparent haptic movement. Perception \& Psychophysics, 1, 175-180.

\section{NOTES}

1. Although the use of equal ISIs separating T1, T2, and T3 is advisable in order to counteract any impact of the tau effect on perceptual reports, this manipulation can inadvertently give rise to undesirable apparent motion effects, especially when ISIs are short. This issue will be further explored in Experiment 2.

2. We thank David Shore for suggesting this manipulation to control for response bias effects.

3. Our thanks to an anonymous reviewer for making this important point and for suggesting the two-alternative forced choice procedure as a tool to find out whether subjective percepts are equivalent on tap and rabbit trials.

4. It might be possible to develop an alternative attentional explanation of the saltation illusion in terms of the involuntary (exogenous) capture of attention by $\mathrm{T} 3$ at $\mathrm{L} 1$ or $\mathrm{L} 3$, even though attention is endogenously focused at L2. However, such an account would clearly be distinct from the explanation of saltation in terms of endogenously mediated states of attention and expectation proposed by Kilgard and Merzenich (1995).

(Manuscript received October 22, 2003; revision accepted for publication June 25, 2004.) 